\title{
DYNAMIC MODELLING OF THE SERIES RESONANT CONVERTER OPERATING IN DISCONTINOUS CONDUCTION MODE AND ITS APPLICATION IN SPACE
}

\author{
A. Soto ${ }^{(1)}$, J. Cortes $^{(1)}$, F. Pascual ${ }^{(1)}$ \\ (l)Airbus Defence and Space, (CRISA), Torres Quevedo 9, 28760 Tres Cantos (Spain), Email:Andres.Soto@airbus.com
}

\begin{abstract}
The Series Resonant Full Bridge operating in Discontinuous Conduction Mode (DCM) at fixed frequency, SRFB-DCM, is an interesting topology to provide isolation in the interface to main buses while having high efficiency thanks to Zero Voltage Switching (ZVS) and Zero Current Switching (ZCS). As this converter cannot be regulated, usually, it is more interesting for regulated buses or in case there is postregulation. This converter finds application in Power Processing Units for Electrical Propulsion and in any secondary high power front-end converter.
\end{abstract}

Despite its simple design without regulation, in general, the dynamic behaviour of resonant converters is difficult to predict. A model is necessary to predict compatibility with input and output interfaces (stability, output impedance, start-up in-rush,...), avoiding late discovery of oscillations, for example.

This paper presents a method to obtain the theoretical dynamic model of the SRFB-DCM with fixed frequency and duty cycle which seems easier to understand but surprisingly not treated in literature. Results are contrasted with simulations.

\section{INTRODUCTION}

A lot of literature can be found about modelling the DC characteristics of the Series Resonant Full Bridge, SRFB, and other resonant converters operating both in Continuous Conduction Mode (CCM) and Discontinuous Conduction Mode (DCM), [1], [2], with frequency/duty as controlling variables, but little about dynamics [3]. This is one of the most difficult topics in power electronics.

The SRFB converter is shown in Fig. 1. All analysis presented in the paper are also valid for half-bridge configuration.

The resonant inductance can be implemented entirely or partially with the leakage inductance of the transformer, thus, the usual problems associated to this inductance are avoided with this topology. This is quite interesting for applications requiring very good isolation between primary and secondary where it is difficult to have a good transformer coupling.

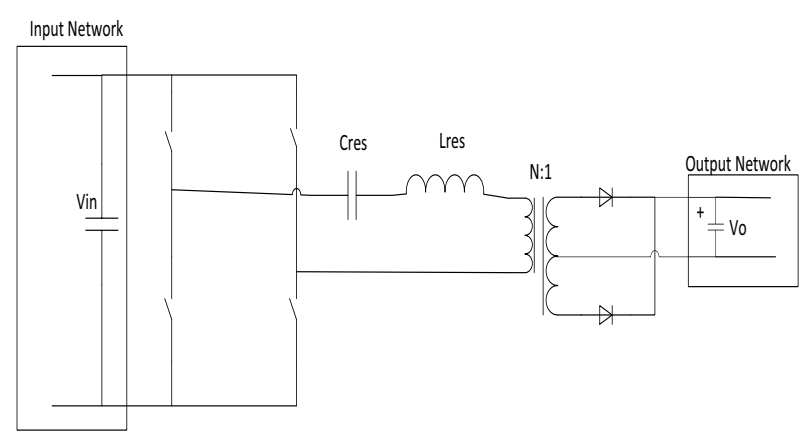

Figure 1. Simplified schematics of SRFB converter.

The SRFB converter working in CCM can only stepdown the input voltage affected by the turns ratio seen in secondary side. The output voltage is regulated commanding the frequency as a function of input voltage and load current. However, regulation becomes difficult or impossible at light loads.

In DCM, the DC transfer function is unity, being output voltage (affected with turns ratio) equal to input voltage independently of the load (assuming no converter losses) and switching frequency as long as DCM operation is maintained. Thus, this converter cannot be regulated.

DCM operation takes place when there is a time before the end of one semi-period, dead time, in which the inductor current keeps constant to zero (or equal to the magnetizing current). As a consequence, the capacitor voltage keeps constant during this dead time.

Two conditions are necessary to obtain DCM behaviour. First, the switching frequency should be lower than the resonant frequency and, second, the maximum absolute resonant capacitor voltage in steady state should be lower than $2 \cdot \widehat{V}_{o}, \widehat{V}_{o}$ is the output voltage affected by the turns ratio.

Thanks to the inductor current dead time, the switches can be turned-off with zero current (ZCS) and the rectifier diodes will not have reverse recovery. It is possible to improve further the efficiency by enabling Zero Voltage Switching (ZVS) having an inductance in parallel with the tank or using the magnetizing current to have a small current in the switches during the dead time. 


\section{FUNDAMENTAL EQUATIONS}

The following assumptions are done to start the analysis of the voltage and current waveforms of the resonant tank operating in DCM:

- Magnetizing current of converter transformer is assumed zero.

- Even in transient conditions, the inductor current at the beginning of the switching period is zero. It could be seen as a strong constraint, but it will be shown it is not the case.

- Input and output voltages, $v_{\text {in }}$ and $\mathrm{v}_{\mathrm{o}}$ are assumed to be constant during a switching semi-period. In other words, input and output switching voltage ripples are small. But the analysis allows for slow changes in these voltages from one period to the other.

- Resistive losses are neglected.

Three first conditions are found easily in practical converters. Resistive losses will be treated later. If magnetizing current is significant with respect to resonant inductor current, then the converter is an LLC resonant type [4]. Here, the model obtained will be valid for converters where the magnetizing current is small compared to main inductor.

Fig. 2 shows a conceptual model of the converter with current and voltage reference which helps to get insight in its operation.

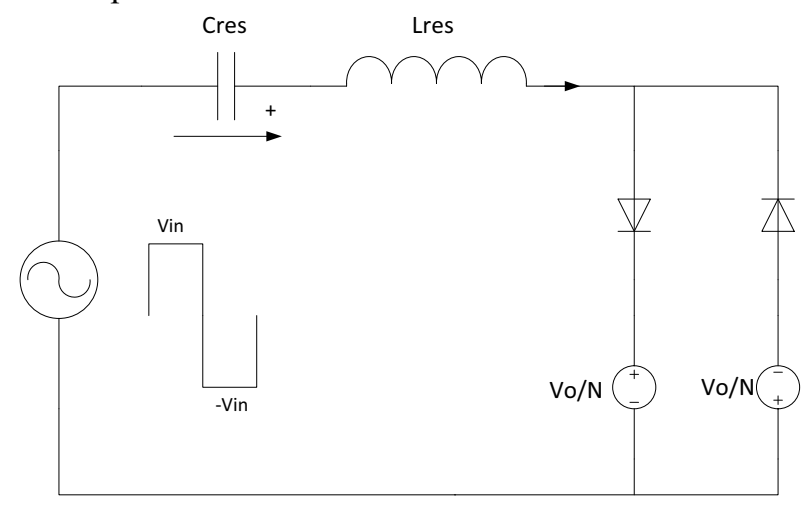

Figure 2. Equivalent simplified circuit and references for voltage and current.

Fig. 3 shows the equivalent circuit for one semi-period. Initial condition for current is zero based on previous assumptions. Initial capacitor voltage $u c 0$ will be obtained as a function of the operating point of the converter.

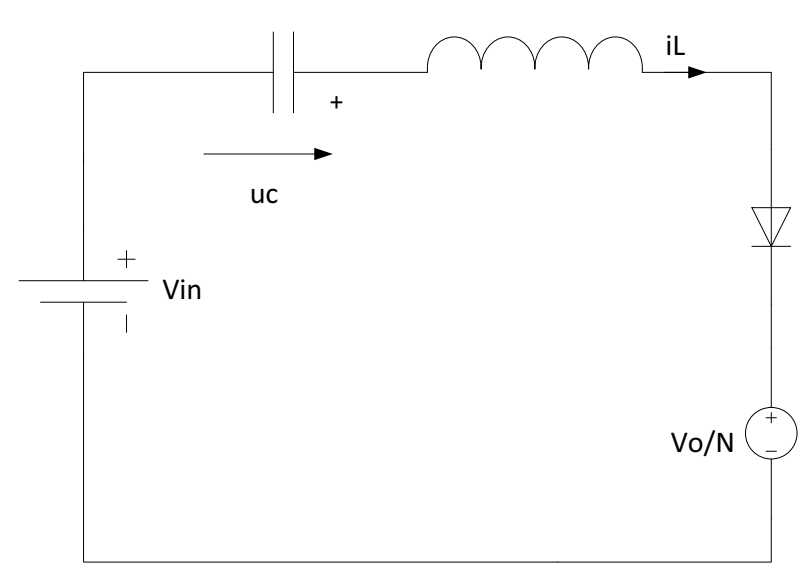

Figure 3. Equivalent circuit for first semi-period (SP1) and references for voltage and current.

The inductor current and capacitor voltage during a switching semi-period, both for transient and steady state operation, have the general forms given by Eq. 1 .

$$
\left[\begin{array}{lrl}
i L(t)=A \cdot \sin \left(\omega_{\text {res }} \cdot t\right), & 0<t \leq \frac{T_{r e s}}{2} \\
i L(t)=0, & \frac{T_{r e s}}{2}<t \leq \frac{T_{s w}}{2}
\end{array}\right.
$$$$
\left[\begin{array}{ll}
u c(t)=A \cdot \cos \left(\omega_{\text {res }} \cdot t\right) \cdot \sqrt{\frac{L_{r e s}}{C_{\text {res }}}}+B, & 0<t \leq \frac{T_{\text {res }}}{2} \\
u c(t)=-A \cdot \sqrt{\frac{L_{r e s}}{C_{\text {res }}}}+B, & \frac{T_{r e s}}{2}<t \leq \frac{T_{s w}}{2}
\end{array}\right.
$$

Constants A and B can be calculated as a function of the initial capacitor voltage $u c 0$ by calculating the current slope at the beginning of the semi-period, as shown in Eq.2. The voltage $\widehat{v_{o}}$ is the output voltage affected by the turns ratio during the switching period. Similarly, vin is the input voltage during the switching period. For the analysis, these two voltages can change slowly from one period to the other, for example during transients.

$$
\begin{aligned}
& {\left[\left.\frac{\operatorname{diL}(t)}{d t}\right|_{t=0}=A \cdot \omega_{\text {res }}=\frac{v_{\text {in }}-\widehat{v_{o}}+u c 0}{L_{\text {res }}}\right.} \\
& u c 0=u c(0)=A \cdot \sqrt{\frac{L_{r e s}}{C_{r e s}}+B} \\
& {\left[\begin{array}{l}
A=\left(v_{\text {in }}-\widehat{v_{o}}+u c 0\right) \cdot \sqrt{\frac{C_{r e s}}{L_{r e s}}} \\
B=-\left(v_{\text {in }}-\widehat{v_{o}}\right)
\end{array}\right.}
\end{aligned}
$$


Merging Eq. 1 and Eq. 2, then Eqs. 3-4 are obtained for one semi-period.

$$
\begin{gathered}
0<t \leq \frac{T_{\text {res }}}{2} \\
i L(t)=\left(v_{\text {in }}-\widehat{v_{o}}+u c 0\right) \cdot \sqrt{\frac{c_{\text {res }}}{L_{\text {res }}}} \cdot \sin \left(\omega_{\text {res }} \cdot t\right) \\
u c(t)=\left(v_{\text {in }}-\widehat{v_{o}}\right) \cdot\left(\cos \left(\omega_{\text {res }} \cdot t\right)-1\right)+u c 0 \cdot \cos \left(\omega_{\text {res }} \cdot t\right) \\
{\left[\begin{array}{c}
\frac{T_{\text {res }}}{2}<t \leq \frac{T_{\text {sw }}}{2} \\
i L(t)=0 \\
u c(t)=-2 \cdot\left(v_{\text {in }}-\widehat{v_{o}}\right)-u c 0
\end{array}\right.}
\end{gathered}
$$

From Eq. 4, it is interesting to notice that during the dead time and at the end of one switching semi-period, the capacitor voltage is the negative of its initial value minus twice the difference between input and output voltage.

The inductor current will not change direction and remain zero leading to a dead time if the voltage applied to the inductor when the other rectifier diodes try to conduct is in the opposite direction. Later in sub-chapter 2.2, this condition for DCM is explored.

The same set of equations Eqs.3-4 are valid in the next semi-period (SP2) if the opposite resonant tank current and voltage are considered, as shown in Fig.4.

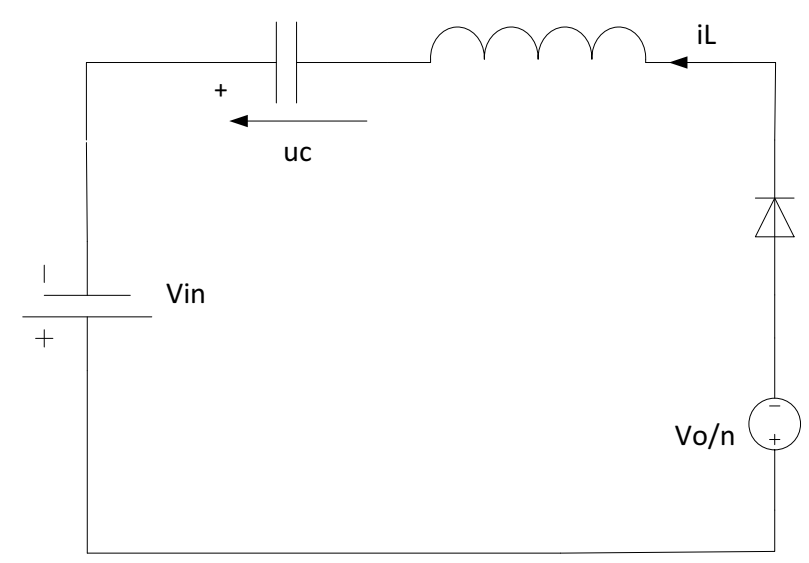

Figure 4. Equivalent circuit for second semi-period (SP2) and opposite references for voltage and current.

The initial condition of the capacitor voltage at each semi-period is the negative of the voltage at the end of the previous semi-period (current is always zero initially), as Eqs. 5-6 show.

$$
\begin{aligned}
& u c_{S P 2}(0)=-u c_{S P 1}\left(\frac{T_{S w}}{2}\right) \\
& u c_{S P 3}(0)=-u c_{S P 2}\left(\frac{T_{S w}}{2}\right)
\end{aligned}
$$

Using the same set of equations given by Eqs. 3-4 for each semi-period with the initial conditions given by Eqs. 5-6, it is possible to create only one model for the different semi-periods where the evolution with time of capacitor voltage and inductor current are given. It is also possible to remove the rectification diodes and the full bridge so the interaction with the rest of the converter is modelled as Fig. 5 shows.

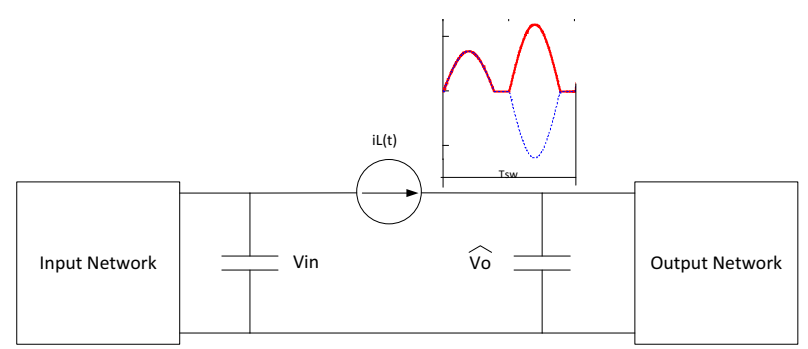

Figure 5. Equivalent model without full bridge and diode rectifiers and definitions of $i l(t), v_{i n}(t)$ and $\widehat{v_{o}}(t)$.

It is important to remark that Eqs. 3-6 are valid for both $\mathrm{DC}$ and $\mathrm{AC}$ analysis. Given an initial condition for the capacitor voltage and the evolution with time of the input and output voltages, it is possible to calculate the evolution of the resonant inductor current, and hence, the dynamics of the converter.

\subsection{Steady state equilibrium}

Steady state in the capacitor can be found only when there is a periodic voltage. So it is necessary that the voltage at the end of the switching period is equal to the value at the beginning.

In the second semi-period (SP2), the initial condition for Eqs. 3-4 is the opposite of $u c_{S P 1}\left(\frac{T_{S W}}{2}\right)$, so at the end of this second semi-period, the end of the switching period, and applying Eq.4, the voltage is given by Eq. 7 .

$$
u c_{S P 2}\left(\frac{T_{S w}}{2}\right)=-4 \cdot\left(V_{\text {in }}-\widehat{V}_{o}\right)-u c 0_{S P 1}
$$

Steady state in the capacitor can be found only when there is a periodic voltage so, given Eq. 6, Eq. 8 should be met.

$$
u c 0_{S P 3}=u c 0_{S P 1}=-u c_{S P 2}\left(\frac{T_{S w}}{2}\right)
$$

Clearly, from Eq. 7 and Eq. 8, it is necessary for periodic voltage that $\mathrm{V}_{\text {in }}=\widehat{V}_{o}$. This is the reason this converter has unity transfer function and why it cannot be controlled with switching frequency or duty if it is operating in DCM. Hereafter, sub-index SP can be removed since both semi-periods have the same waveforms. 
On the other hand, converter will be in steady state when the rectified average inductor current is equal to average load current $\widehat{I}_{o}$, referred to primary, as seen in Fig. 5. Then, considering that $\mathrm{V}_{\text {in }}=\widehat{V}_{o}$ and that the current waveform is the same for the two semi-periods, it follows Eq. 9.

$$
\begin{gathered}
<i L(t) \geq \frac{1}{T_{s w}} \cdot \int_{0}^{T_{s w}} i L(t) \cdot d t= \\
=\frac{1}{\frac{T_{s w}}{2}} \cdot \int_{0}^{\frac{T_{r e s}}{2}} u c 0 \cdot \sqrt{\frac{C_{r e s}}{L_{r e s}}} \cdot \sin \left(\omega_{\text {res }} \cdot t\right) \cdot d t
\end{gathered}
$$

Eq.9 leads to the equality given in Eq. 10 .

$$
\widehat{I}_{o}=<i L(t)>=\frac{2}{\pi} \cdot \frac{f s w}{f r e s} \cdot u c 0 \cdot \sqrt{\frac{c_{r e s}}{L_{r e s}}}
$$

The initial resonant capacitor voltage as a function of load current at each semi-period in steady state is then given by Eq. 11 .

$$
u c 0=\widehat{I_{o}} \cdot \sqrt{\frac{L_{r e s}}{c_{r e s}}} \cdot \frac{\pi}{2} \cdot \frac{\text { fres }}{f s w}
$$

\subsection{Conditions for DCM}

It is interesting to have a way to determine when the converter could lose DCM, and then soft-switching. It is desirable this criteria is valid not only for steady state but also for transient operation.

The inductor current will not change direction during a semi-period and remain zero if the resulting voltage applied to the inductor when the current tries to change direction is opposing to this change, as given in Eq. 12.

$$
\begin{gathered}
-\left(-2 \cdot\left(v_{i n}-\widehat{v_{o}}\right)-u c 0\right)-\left(v_{i n}+\widehat{v_{o}}\right)<0 \\
u c 0<3 \cdot \widehat{v_{o}}-v_{i n}
\end{gathered}
$$

If the capacitor voltage at the beginning of a semiperiod is meeting Eq.12, then the converter stays in DCM. This condition is valid for steady state and transient operation.

In steady state, using Eqs. $11-12$ and $\mathrm{V}_{\text {in }}=\widehat{V}_{o}$, then it is obtained Eq. 13.

$$
\sqrt{\frac{L_{r e s}}{C_{r e s}}} \cdot \frac{\pi \cdot f r e s}{4 \cdot f s w} \cdot \widehat{I}_{o}<\widehat{V}_{o}
$$

Clearly, there is a maximum current allowed to stay in DCM.

Only in especial transients Eq.12 could not be met. For example, during starting-up or short-circuit events.

\section{TRANSIENT MODELLING}

Based on Eq. 3-4, it is possible to derive a time-discrete model for the averaged model where the switching is removed.

Being $k$ an index to refer to each semi-period, the time evolution of current and voltage during that semi-period is given by Eq. 14, where $t=0$ is the beginning of that semi-period.

$$
\begin{gathered}
0<t \leq \frac{T_{\text {res }}}{2} \\
i L(t)_{k}=\left(v_{\text {in }}(k)-\widehat{v_{o}}(k)+u c 0(k)\right) \cdot \sqrt{\frac{C_{\text {res }}}{L_{\text {res }}}} \cdot \sin \left(\omega_{\text {res }} \cdot t\right) \\
u c(t)_{k}=\left(v_{\text {in }}(k)-\widehat{v_{o}}(k)+u c 0(k)\right) \cdot\left(\cos \left(\omega_{\text {res }} \cdot t\right)-1\right)+u c 0(k) \\
{\left[\begin{array}{l}
\frac{T_{\text {res }}}{2}<t \leq \frac{T_{\text {sw }}}{2} \\
i L(t)_{k}=0 \\
u c(t)_{k}=-2 \cdot\left(v_{\text {in }}(k)-\widehat{v_{o}}(k)\right)-u c 0(k)
\end{array}\right.}
\end{gathered}
$$

The capacitor voltage evolution at the beginning of each semi-period can be described, based on Eqs. 3-6, as the sequence given in Eq. 15.

$$
u c 0(k+1)=2 \cdot\left(v_{i n}(k)-\widehat{v_{o}}(k)\right)+u c 0(k)
$$

The investigated dynamic model should replace the sinusoidal current of the inductor by an averaged current value depending on $\mathrm{k}$, iL $(\mathrm{k})$, that provides the same dynamic response. Particularly, iL(k) evolution should provide the same charge transfer from input to output at each semi-period.

Looking at Fig. 5, the charge transferred from input to output during semi-period $k$ is given by Eq. 16 using Eq. 14.

$$
\begin{aligned}
& Q(k)=\int_{\frac{T_{S W}}{2} \cdot k}^{\frac{T_{S W}}{2} \cdot(k+1)} i L(t) \cdot d t=\int_{0}^{\frac{T_{S W}}{2}} i L(t)_{k} \cdot d t= \\
& =\frac{1}{\pi} \cdot \frac{1}{\text { fres }} \cdot \sqrt{\frac{C_{\text {res }}}{L_{\text {res }}}} \cdot\left(v_{\text {in }}(k)-\widehat{v_{o}}(k)+u c 0(k)\right)
\end{aligned}
$$

This charge is a function of $\mathrm{V}_{\text {in }}(\mathrm{k}), \widehat{v_{o}}(\mathrm{k})$ and $\mathrm{uc} 0(\mathrm{k})$, which is also a function of input and output voltages as per Eq. 15. Then, Eq. 15 and Eq.16 form a system of equation that should explain the dynamics of the whole converter.

Current $\mathrm{iL}(\mathrm{k})$ could be understood as a constant current during the semi-period that gives the charge calculated in Eq. 16, so Eq.17 is obtained.

$$
Q(k)=i L(k) \cdot \frac{T s w}{2}
$$


It would mean that from semi-period to semi-period, the current that is being modelled is transiently evolving in steps, which has no physical meaning. Actually, if going this way, a resistor is found in the averaged model which introduces losses. But the analysed converter is ideal with no losses, so the model cannot be fully correct.

Instead, if a linear variation of averaged current is considered during a semi-period and if $\mathrm{iL}(\mathrm{k})$ is the averaged current at the beginning of the semi-period $\mathrm{k}$, then Eq. 18 is obtained.

$$
Q(k)=\left(i L(k)+\frac{i L(k+1)-i l(k)}{2}\right) \cdot \frac{T s w}{2}
$$

Then, merging Eqs. 15-16 and Eq. 18, the system of equations to solve is given by Eq. 19 .

$\left[\begin{array}{l}i L(k)+\frac{i L(k+1)-i L(k)}{2}=\frac{2}{\pi} \cdot \frac{f s w}{f r e s} \cdot\left(v_{\text {in }}(k)-\widehat{v_{o}}(k)+u c 0(k)\right) \cdot \sqrt{\frac{c_{\text {res }}}{L_{\text {res }}}} \\ u c 0(k+1)=2 \cdot\left(v_{\text {in }}(k)-\widehat{v_{o}}(k)\right)+u c 0(k)\end{array}\right.$

Doing some maths to remove uc0(k) from the system of equations and solve for iL(k), Eq. 20 is obtained.

$$
\begin{gathered}
\frac{i L(k+2)-i L(k)}{2}= \\
=\frac{2}{\pi} \cdot \frac{f s w}{\text { fres }} \cdot \sqrt{\frac{C_{\text {res }}}{L_{\text {res }}}} \cdot\left(v_{\text {in }}(k+1)-\widehat{v_{o}}(k+1)+v_{\text {in }}(k)-\widehat{v_{o}}(k)\right)
\end{gathered}
$$

Eq. 20 gives the evolution of the current only as a function of the input and output voltages. This equation could be solved numerically with Tsw/2 as time step together with input and output networks to obtain the dynamics of the converter.

This time-discrete equation does not resemble an equation with a known solution. Doing more maths with operators, being the operator $\Delta$ defined as $\Delta \cdot x(k)==$ $\frac{x(k+1)-x(k)}{T s}$ with $T s=\frac{1}{2 \cdot f_{s w}}$, then Eqs. 21-23 are obtained.

$$
\begin{gathered}
\frac{T_{s}^{2}}{2} \cdot \Delta^{2} \cdot i L(k)+T_{s} \cdot \Delta \cdot i L(k)= \\
=\frac{4}{\pi} \cdot \frac{f s w}{\text { fres }} \cdot \sqrt{\frac{C_{r e s}}{L_{r e s}}} \cdot\left[\frac{T_{s}}{2} \cdot \Delta+1\right] \cdot\left(v_{\text {in }}(k)-\widehat{v_{o}}(k)\right) \\
=\frac{4}{\pi} \cdot \frac{f s w}{\text { fres }} \cdot \sqrt{\frac{T_{s}}{L_{r e s}}} \cdot\left[\frac{T_{s}}{2} \cdot \Delta+1\right] \cdot\left(v_{\text {in }}(k)-\widehat{v_{o}}(k)\right) \\
\Delta \cdot i L(k)=\frac{8}{\pi} \cdot \frac{f s w^{2}}{\text { fres }} \cdot \sqrt{\frac{C_{r e s}}{L_{r e s}}} \cdot\left(v_{\text {in }}(k)-\widehat{v_{o}}(k)\right)
\end{gathered}
$$

Now it is easy to recognise in Eq. 23 the time discrete form of a derivative. Assuming slow change of input and output voltages during one semi-period, assumption already done for the derivation, then this expression can be put in continuous time as in Eq. 24 .

$$
\frac{d i L(t)}{d t}=\frac{8}{\pi} \cdot \frac{f s w^{2}}{\text { fres }} \cdot \sqrt{\frac{C_{r e s}}{L_{r e s}}} \cdot\left(v_{\text {in }}(t)-\widehat{v_{o}}(t)\right)
$$

Eq. 24 is the equation of an inductor with inductance value given by Eq. 25 .

$$
L_{e q}=\frac{\pi}{8} \cdot \frac{f r e s}{f s w^{2}} \cdot \sqrt{\frac{L_{r e s}}{C_{r e s}}}=\frac{1}{16} \cdot \frac{1}{f s w^{2}} \cdot \frac{1}{C_{r e s}}
$$

Remarkably, the equivalent inductance value depends only on the selection of the resonant capacitance once the switching frequency is fixed.

Fig. 6 shows the averaged model obtained so far replacing in Fig. 5 the resonant current source by the inductor just obtained.

$$
L_{\text {eq }}=\frac{\pi}{8} \cdot \frac{f r e s}{f s w^{2}} \cdot \sqrt{\frac{L_{\text {res }}}{C_{\text {res }}}}
$$

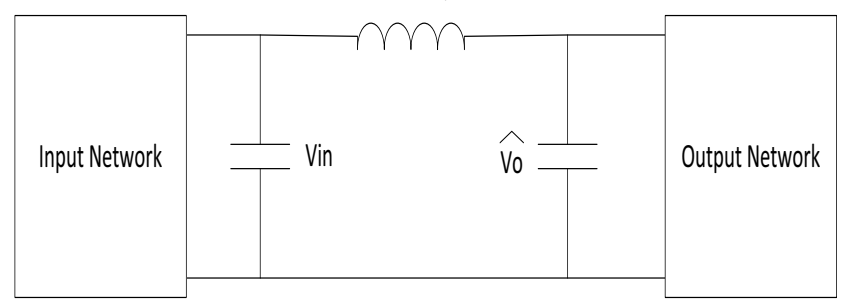

Figure 6. Equivalent dynamic averaged model for the SRFB converter in DCM.

The evolution of the resonant capacitor voltage, although not necessary to know for the dynamics of the converter, can be also calculated easily now. It is important to have it to know the stress of the capacitor and to know if the hypothesis of DCM is kept.

Using $\Delta$ operator defined before, Eq. 15 becomes Eq. 26 .

$$
\Delta \cdot u c 0(k)=4 \cdot f s w \cdot\left(v_{i n}(k)-\widehat{v_{o}}(k)\right)
$$

From Eq.23, it is possible to obtain Eq.27.

$$
\begin{aligned}
& \Delta \cdot u c 0(k)=4 \cdot f s w \cdot L e q \cdot \Delta \cdot i L(k) \\
& u c 0(k)=4 \cdot f s w \cdot L e q \cdot i L(k)+C
\end{aligned}
$$

Constant $\mathrm{C}$ in Eq.27 is determined forcing that in steady state Eq. 11 is met, so $\mathrm{C}$ becomes 0. Then, Eq. 27 is showing that the initial value of the capacitor voltage at each semi-period is proportional to averaged inductor current. 


\subsection{Modelling of losses and damping}

So far, it has been assumed no resistive voltage drop in the resonant tank. The approximation is very good to calculate the waveforms of current and capacitor. However, since this assumption implies no resistive behavior in the power path (no Rdson, ESR, inductor resistance,...), it fails to predict the losses of the converter and its damping, as comparison with simulations and test shows.

The approach that is followed to calculate the equivalent resistance is more heuristic but quite effective, as seen later in the simulation verification and also seen in tests verification.

The real total resistance of the topology cannot predict correctly the losses in an average model since the actual currents are much higher due to its sinusoidal shape than the current predicted by the average model.

One method to obtain the equivalent resistance is to make the losses of the averaged model equal to the losses in the switched converter. It means Eq. 28 should hold.

$$
<i L^{2}(t)>\cdot \text { Rreal }=<i L(t)>^{2} \cdot R_{e q}
$$

Doing some calculations, the equivalent resistance given by Eq. 29 can be found.

$$
R_{e q}=\frac{\pi^{2}}{8} \cdot \frac{\text { fres }}{f s w} \cdot \text { Rreal }
$$

The final model is shown in Fig. 7. No small signal assumption has been done during the analysis so this model should be valid also for large transients, as following chapter will show.

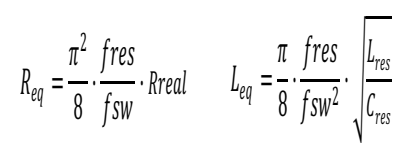

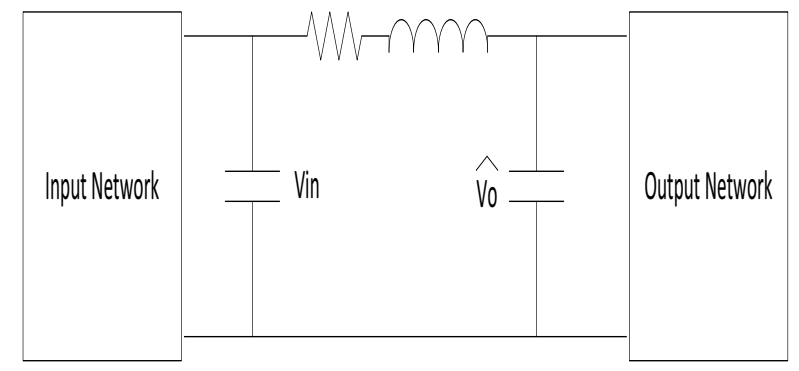

Figure 7. Equivalent averaged model with dynamics and losses.

\section{SIMULATIONS RESULTS}

A simulator tool able to do frequency analysis of switched converters is needed. Simplis simulator is used since it is possible to obtain the frequency response from a switched time simulation, as if measuring the loop gain of a converter in the lab.

The simulated converter, Fig.8, does not have input filter neither output filter for simplification. The main parameters are $\mathrm{R}_{\text {real }}=60 \mathrm{~m} \Omega, \mathrm{L}_{\text {res }}=1 \mu \mathrm{H}, \mathrm{C}_{\text {res }}=1.5 \mu \mathrm{F}$, $\mathrm{f}_{\mathrm{sw}}=100 \mathrm{kHz}, \mathrm{V}_{\text {in }}=100 \mathrm{~V}, \mathrm{~N}=1.5$ and $\mathrm{C}_{\mathrm{o}}=500 \mu \mathrm{F}$. Diodes and MOSFETs are modelled as ideal elements to simplify the comparison.

The output impedance comparison in frequency domain, Fig. 9, shows a perfect matching of the model with simulations results. The output impedance comparison in time domain for large signal (5 A load step) is shown in Fig.10. A perfect matching both in DC and AC can be seen.
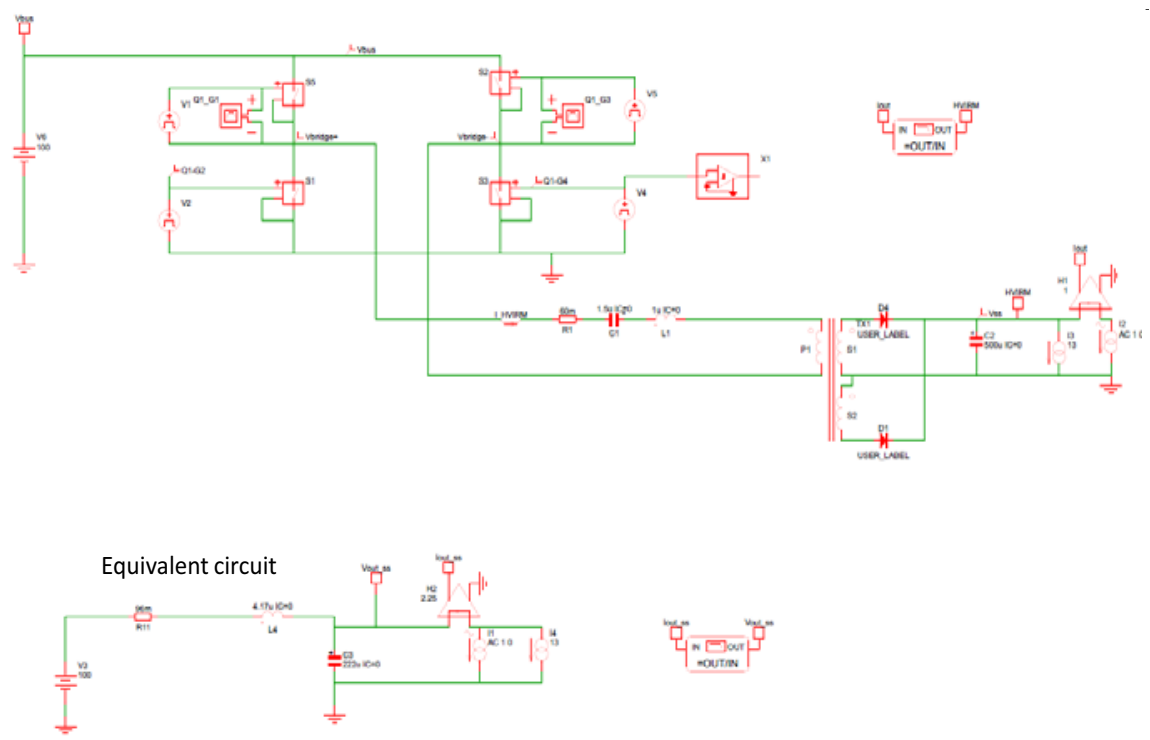

Figure 8. Switched model and equivalent averaged model used in Simplis simulator for model verification. 


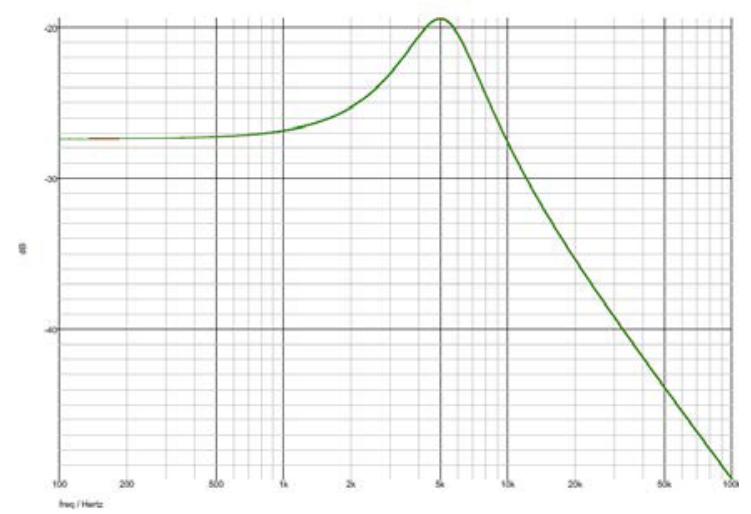

Figure 9. Output impedance frequency response comparison: green switched converter with Simplis, red averaged model.

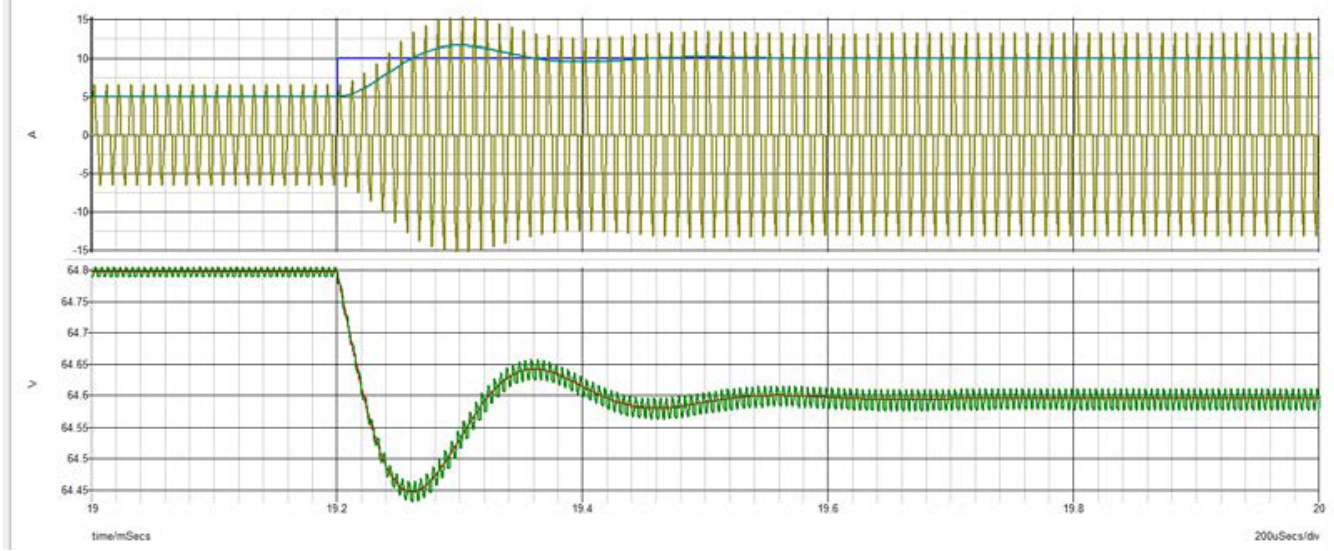

Figure 10. Load step large signal transient. Lower plot: output voltage response, green switched model, red averaged model. Upper plot: inductor current and load step, yellow switched model, cyan averaged model.

\section{CONCLUSIONS}

The paper derives a dynamic theoretical model for the Series Resonant Converter Full Bridge operating in DCM. This converter has many applications due to its simplicity and high efficiency, especially as front-end converter providing isolation and interfacing regulated buses or un-regulated buses with post-regulations. Despite its simplicity and not being a regulated converter, it is necessary to know its dynamics to design and justify properly the converter interfacing the input and output networks.

This paper obtains a simple model with only one resistor and inductance. Theoretical expressions are given for these elements which enable design-oriented analysis. Paradoxically, being the DCM series resonant converter operated below resonance, capacitive behaviour, the equivalent model is an inductor of value depending exclusively on the resonant capacitance and switching frequency.

The model has been validated with simulation results, showing a perfect prediction both for small-signal analysis and also for large transients.
The model has been used to design and justify properly the input and output filters of a flight converter and also the stability with its load.

\section{REFERENCES}

1. Erickson, R., Maksimovic, D. Fundamental of Power Electronics, Second Edition, 2001 University of Colorado, Boulder, Colorado. pp 706-759.

2. Vorperian, V., Cuk, S., A Complete DC Analysis of the Series Resonant Converter, IEEE Power Electronics Specialist Conference 1982, pp. 85-100

3. Witulski, F., Hernándes, A. and Erickson, R. Small Signal Equivalent Circuit Modeling of Resonant Converters, IEEE Transactions on Power Electronics, Vol. 6, No. 1 January 1991.

4. Lazar, J.F., Martinelli, R. Steady-state analysis of the LLC series resonant converter, IEEE Applied Power Electronics Conference and Exposition 2001 